\title{
Wintterlin, Florian: Quelle: Internet. Journalistisches Vertrauen bei der Recherche in sozialen Medien
}

\author{
Baden-Baden: Nomos 2019. 280 Seiten. Preis: $€ 54$
}

\section{Uwe Krüger}

Online publiziert: 17. März 2020

(C) Der/die Autor(en) 2020

Vertrauensforschung boomt in der deutschen Kommunikationswissenschaft, spätestens seit den „Lügenpresse“-Rufen in Dresden ab 2014. Einer der Orte, an denen schon vorher über Medienvertrauen nachgedacht wurde, ist die Westfälische Wilhelms-Universität Münster. In derem interdisziplinären Graduiertenkolleg „Vertrauen und Kommunikation in einer digitalisierten Welt" ist auch die Dissertation von Florian Wintterlin entstanden.

Wintterlin untersucht nicht das Medienvertrauen von RezipientInnen, aber einen mutmaßlich damit zusammenhängenden bzw. vorgelagerten Faktor, nämlich das Vertrauen von JournalistInnen in ihre Quellen. In der Ära digitaler Netzwerkmedien, so die Prämisse seiner Arbeit, steht der Journalismus zunehmend unter Druck, die inflationäre Vermehrung und Beschleunigung von Kommunikation angemessen zu verarbeiten, ohne dass Korrektheit, Objektivität und Unparteilichkeit der Berichterstattung beeinträchtigt werden. An Bedeutung gewinnen nun „distanzierte Quellen“, also Akteure, die die JournalistInnen nicht persönlich getroffen haben, sondern deren „Inhalte über Social Media recherchiert und in die Nachrichten eingebunden“ werden (S. 17), und zwar besonders in der Krisenberichterstattung.

In der Dissertation wird das Verhältnis von JournalistInnen zu diesen „distanzierten Quellen“ theoretisch konzeptualisiert und in drei aufeinander aufbauenden empirischen Studien untersucht. Zunächst führt Wintterlin eine deskriptive Inhaltsanalyse der deutschen Berichterstattung über acht politische Krisen, Naturkatastrophen und Terroranschläge zwischen 2011 und 2015 durch. In dem Sample aus 761 Online-, TV-, Print- und Radiobeiträgen aus Leitmedien wurde demnach Social-Media-Material ,in nicht zu vernachlässigendem Umfang als Quelle genutzt“ (S. 63),

\footnotetext{
Dr. U. Krüger $(\bowtie)$

Institut für Kommunikations- und Medienwissenschaft, Universität Leipzig, Nikolaistr. 27-29, 04109 Leipzig, Deutschland

E-Mail: uwe.krueger@uni-leipzig.de
} 
am häufigsten von Online-Medien, am häufigsten direkt nach dem Ausbruch einer Krise und im Krisen-Vergleich am häufigsten beim Thema Syrien.

Wie Redaktionen mit Unsicherheiten bezüglich „distanzierter Quellen“ umgehen und welche Rolle Vertrauen dabei spielt, klärt Wintterlin dann in qualitativen Experteninterviews: Zwölf JournalistInnen, vom Social-Media-Redakteur eines Printmagazins bis zum Nachrichtenchef einer Nachrichtenagentur, geben Einblicke in ihre Arbeit mit sozialen Netzwerkplattformen, in ihre Abwägungsprozesse und Verifikationsstrategien. Auf Grundlage dieser Interviews entwickelt Wintterlin ein „Modell reflexiven Vertrauens in distanzierte Quellen“, in dem die „Antezedenzien von Risikowahrnehmung und Vertrauenswürdigkeit aus Sicht von JournalistInnen“ (S. 119, 183) versammelt sind. Neu daran gegenüber der existierenden Literatur ist der Faktor der Risikowahrnehmung: „Wenn die Risikowahrnehmung niedrig ist, nehmen JournalistInnen Abstand von der zeitaufwändigen Verifikation und wählen die eher pragmatische Strategie, distanzierte Quellen wegen ihrer einfachen Verfügbarkeit zu verwenden." (S. 184)

Zuletzt führt der Autor eine standardisierte Online-Befragung von 411 JournalistInnen aus Deutschland und Großbritannien (das europaweit führende Land bei der journalistischen Social-Media-Verifikation) durch. Er will wissen, wie JournalistInnen in der Breite mit Social-Media-Material umgehen und von welchen Faktoren ihre Beurteilung von Vertrauenswürdigkeit und Risiko in der Arbeit mit ,distanzierten Quellen“ abhängt. Die Ergebnisse zeigen keine signifikanten Unterschiede zwischen Deutschen und Briten. Erklärkraft für Unterschiede in den unabhängigen Variablen haben eher der organisationale Hintergrund, das Social-Media-Wissen und die epistemologischen Überzeugungen der JournalistInnen.

Florian Wintterlin hat eine originelle und vor allem in empirischer Hinsicht starke Dissertation vorlegt, die den vorliegenden Forschungsstand zum Sourcing und zum Verhältnis zwischen JournalistInnen und Quellen bereichert. Er macht auch deutlich, dass seine Befragungsergebnisse nicht repräsentativ, sondern explorativ zu sehen sind und zudem von sozialer Erwünschtheit verzerrt sein könnten - denn wer gibt schon gern zu, Quellen ungeprüft zu übernehmen. Verdienstvoll ist ebenfalls, dass er die soziologische Literatur zu Vertrauen und zum Umgang mit Unsicherheit für die Sourcing-Forschung fruchtbar macht.

Einige Schwächen sehe ich jedoch in der Begriffs- und Theoriearbeit. So wird der zentrale Begriff „,distanzierte Quellen“ definiert als Akteure, „denen JournalistInnen nicht persönlich begegnen können oder wollen“ (S. 252). Unklar bleibt aber, ob es ausreicht, dass ,,deren Inhalte hauptsächlich über Social-Media-Kanäle recherchiert werden“ (S. 130) oder ob die JournalistInnen diese räumlich von ihnen getrennten Quellen auch zwingend ,über Social Media kontaktieren“ (S. 209) oder ,über digitale Kanäle“ kontaktieren (S. 18) (also auch per E-Mail?) oder generell mit ihnen ,interagieren“ (S. 257) (also auch per Telefon?). Theoretisch fußt die Arbeit über weite Strecken auf systemtheoretischen Überlegungen von Blöbaum und Kohring. Für die letzte Teilstudie wird noch überraschend Bourdieus Feldtheorie eingeführt, diese aber für die quantitative Befragung wenig überzeugend operationalisiert (Epistemologien der JournalistInnen werden da zu einer Variable ihres „,kulturellen Kapitals“ und ihre Ressortzugehörigkeit zu einer Variable ihres „ökonomischen Kapitals“). So bleibt diese kritische Gesellschaftstheorie seltsam 
unverbunden mit dem Rest der Arbeit. Zugleich bleiben Theorien mittlerer Reichweite, die macht- und ideologiekritisch das Sourcing im Journalismus adressieren (Indexing-Hypothese, Propagandamodell), unerwähnt.

Generell zeigt sich Wintterlin eher an formalen als an inhaltlichen Aspekten der Nutzung ,distanzierter Quellen“ interessiert. Dabei hätte die Frage ja nahegelegen (und durch Inhaltsanalyse beantwortet werden können), ob etwa in der Syrien- oder Ukraine-Berichterstattung das Informations- und Meinungsspektrum durch SocialMedia-Quellen erweitert worden ist oder ob nur solche Tweets in Artikel eingebunden wurden, die in die dominanten Narrative und Frames des medialen Mainstreams passten - und vielleicht deshalb den JournalistInnen vertrauenswürdig erschienen waren.

Um mit Bourdieu zu sprechen: Mit dieser Arbeit kann Florian Wintterlin im wissenschaftlichen Feld Kapital sammeln als versierter Empiriker, der verschiedene Erhebungsmethoden und elaborierte statistische Auswertungsverfahren beherrscht. Allerdings ist eine Art von Wissen herausgekommen, die nur für einen kleinen Kreis von KollegInnen interessant, aber weder für die Berufspraxis besonders nützlich noch für eine breitere medienkritische Öffentlichkeit relevant ist.

Funding Open Access funding provided by Projekt DEAL.

Open Access Dieser Artikel wird unter der Creative Commons Namensnennung 4.0 International Lizenz veröffentlicht, welche die Nutzung, Vervielfältigung, Bearbeitung, Verbreitung und Wiedergabe in jeglichem Medium und Format erlaubt, sofern Sie den/die ursprünglichen Autor(en) und die Quelle ordnungsgemäß nennen, einen Link zur Creative Commons Lizenz beifügen und angeben, ob Änderungen vorgenommen wurden.

Die in diesem Artikel enthaltenen Bilder und sonstiges Drittmaterial unterliegen ebenfalls der genannten Creative Commons Lizenz, sofern sich aus der Abbildungslegende nichts anderes ergibt. Sofern das betreffende Material nicht unter der genannten Creative Commons Lizenz steht und die betreffende Handlung nicht nach gesetzlichen Vorschriften erlaubt ist, ist für die oben aufgeführten Weiterverwendungen des Materials die Einwilligung des jeweiligen Rechteinhabers einzuholen.

Weitere Details zur Lizenz entnehmen Sie bitte der Lizenzinformation auf http://creativecommons.org/ licenses/by/4.0/deed.de.

Dr. Uwe Krüger ist wissenschaftlicher Mitarbeiter am Institut für Kommunikations- und Medienwissenschaft der Universität Leipzig. 